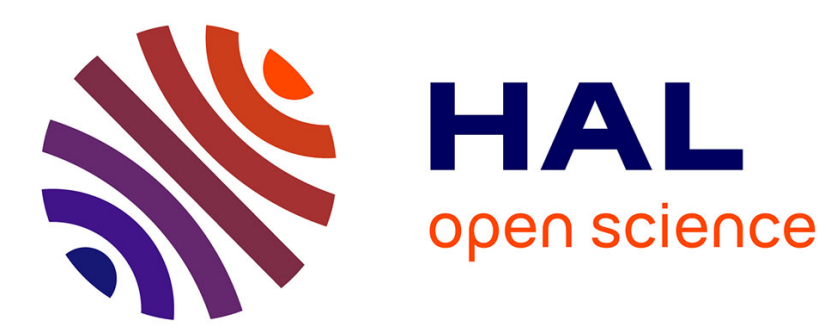

\title{
Cryostat isolant à joints froids démontables: application au stator d'une machine supraconductrice
}

\author{
Y. Brunet, P. Gianese, Pascal Tixador
}

\section{To cite this version:}

Y. Brunet, P. Gianese, Pascal Tixador. Cryostat isolant à joints froids démontables : application au stator d'une machine supraconductrice. Revue de Physique Appliquée, 1988, 23 (11), pp.1773-1776. 10.1051/rphysap:0198800230110177300 . jpa-00246007

\section{HAL Id: jpa-00246007 https://hal.science/jpa-00246007}

Submitted on 1 Jan 1988

HAL is a multi-disciplinary open access archive for the deposit and dissemination of scientific research documents, whether they are published or not. The documents may come from teaching and research institutions in France or abroad, or from public or private research centers.
L'archive ouverte pluridisciplinaire HAL, est destinée au dépôt et à la diffusion de documents scientifiques de niveau recherche, publiés ou non, émanant des établissements d'enseignement et de recherche français ou étrangers, des laboratoires publics ou privés. 


\title{
Cryostat isolant à joints froids démontables : application au stator d'une machine supraconductrice
}

\author{
Y. Brunet, P. Gianese et P. Tixador \\ Centre de Recherche sur les Très Basses Températures, C.N.R.S., B.P. 166 X, 38042 Grenoble Cedex, France
}

(Reçu le 11 avril 1988, accepté le 13 juillet 1988)

\begin{abstract}
Résumé. - L'utilisation de matériaux supraconducteurs à l'induit des machines électriques nécessite la réalisation de cryostats Hélium non conducteurs électriques. Un cryostat horizontal de ce type a été réalisé. Il permet le passage d'un rotor de diamètre $180 \mathrm{~mm}$ et est entièrement démontable. Les essais cryogéniques ont montré que le comportement à froid était satisfaisant et ne nécessitait pas de pompage permanent. Les pertes du cryostat, en fonctionnement autonome, sont de $4 \mathrm{~W}$.

Abstract. - The use of superconducting materials for the armature windings of electrical machines needs non conducting materials for the Helium vessel. Such a cryostat has been designed and built with a $180 \mathrm{~mm}$ horizontal bore for the rotor windings. It remains easy to take it down into pieces as special joints have been set up. Cryogenic tests have shown that our type of cryostat is efficient at low temperature during permanent running even with no extra permanent pumping. Heat losses are lower than $4 \mathrm{~W}$. The cryostat has been used to test a $20 \mathrm{kVA}$, three phase armature winding made with low loss superconducting materials.
\end{abstract}

Les matériaux utilisables à la fréquence industrielle [1] $-50 \mathrm{~Hz}$ - rendent nécessaire la réalisation de cryostats ne présentant pas (ou peu) de pertes en champ magnétique variable. Cette solution est indispensable dans toutes les parties actives d'une machine électrique dont le rotor et le stator seraient supraconducteurs, puisque des champs tournants apparaissent dans tout l'espace environnant les enroulements.

Dans le but de réaliser une machine entièrement supraconductrice de $20 \mathrm{kVA}$ un cryostat horizontal a été conçu et construit : il contient des enroulements statoriques triphasés $50 \mathrm{~Hz}$ et permet le passage d'un rotor adapté. L'accès des bobinages est assuré grâce à la réalisation d'une enceinte à Hélium liquide démontable.

\section{Principes généraux.}

Soumis à un champ magnétique variable, les enceintes contenant les enroulements doivent être isolantes électriquement, dans leurs parties centrales, notamment. Seuls, quelques éléments de faible volume peuvent être conducteurs : une tige en acier inoxydable $\varnothing=4 \mathrm{~mm}, L=40 \mathrm{~mm}$ est le siège de $6 \mathrm{~mW}$ de pertes dans un champ transversal de $0,2 \mathrm{~T} ; 50 \mathrm{~Hz}$. Hors du champ magnétique, des raccords métalli- ques sont possibles. L'ensemble doit être résistant mécaniquement pour permettre la transmission des efforts électrodynamiques et comporter un trou central pour laisser le passage à un inducteur tournant, normal ou supraconducteur.

Notre choix s'est porté sur la résine époxy (araldite CIBA CY 230 + durcisseur HY 951) associée à la fibre de verre (Roving 92140 et 92110 VOSSCHEMIE) dont les propriétés sont données dans le tableau I.

Ce type de composite présente de nombreux avantages [5-6] :

- isolant électrique et thermique excellent,

- matériaux de mise en cuvre aisée, même de façon « artisanale »,

- assemblage facile, par collage, même avec des matériaux métalliques.

Néanmoins son emploi dans un environnement cryogénique reste délicat [5-6] :

- son émissivité élevée induit des pertes, par rayonnement importantes, si on ne prend pas de précautions particulières,

- sa faible diffusivité thermique et son enthalpie importante entraînent des mises en équilibre thermique lentes, 
Tableau I. - Propriétés du matériau composite époxy-Fibre de verre. [Properties of epoxy Fibre composite.]

\begin{tabular}{|c|c|c|c|c|c|c|c|}
\hline & $\begin{array}{l}\text { Conduct. } \\
\text { thermique }\end{array}$ & $\begin{array}{l}\text { Chaleur } \\
\text { spécifique }\end{array}$ & $\begin{array}{l}\text { Diffusivité } \\
\text { thermique }\end{array}$ & $\begin{array}{l}\text { Module } \\
\text { d'Young }\end{array}$ & $\begin{array}{l}\text { Contrainte } \\
\text { max. de } \\
\text { traction }\end{array}$ & $\begin{array}{c}\text { Contrainte } \\
\text { max. de } \\
\text { compression }\end{array}$ & $\begin{array}{c}\text { Contrainte } \\
\text { max. de } \\
\text { cisaillement }\end{array}$ \\
\hline $\begin{array}{c}T \\
(\mathbf{K})\end{array}$ & $\begin{array}{c}K \\
(\mathrm{~W} / \mathrm{mK}) \\
{[2]}\end{array}$ & $\begin{array}{c}C \\
(\mathrm{~J} / \mathrm{kgK}) \\
{[3]}\end{array}$ & $\begin{array}{c}D \\
\left(10^{-6} \mathrm{~m}^{2} / \mathrm{s}\right) \\
\cdot[2]\end{array}$ & $\begin{array}{c}E \\
(\mathrm{GPa}) \\
{[4]}\end{array}$ & $\begin{array}{c}\sigma_{\mathrm{t}} \\
\left(\mathrm{N} / \mathrm{mm}^{2}\right) \\
{[4]}\end{array}$ & $\begin{array}{c}\sigma_{\mathrm{c}} \\
\left(\mathrm{N} / \mathrm{mm}^{2}\right) \\
{[4]}\end{array}$ & $\begin{array}{c}\tau \\
\left(\mathrm{N} / \mathrm{mm}^{2}\right) \\
{[4]}\end{array}$ \\
\hline 300 & 0,89 & 1935 & 0,5 & 26 & 550 & 290 & 9 \\
\hline 80 & 0,3 & 180 & 0,5 & & 950 & 695 & 21 \\
\hline 5 & 0,05 & 1,19 & 10 & 31,6 & 850 & 710 & 18 \\
\hline
\end{tabular}

- les faibles coefficients de dilatation rendent délicates les liaisons avec d'autres matériaux.

- Diffusion gazeuse à travers les parois, si leur épaisseur est faible.

Pour éviter certains de ces inconvénients, nous avons choisi d'utiliser des épaisseurs de parois suffisantes $(>2 \mathrm{~mm})$, d'améliorer les états de surface par un vernis et d'éviter l'emploi de composites pour les retours d'hélium gaz par exemple. Les pertes sont diminuées en complétant l'isolation thermique avec du superisolant à film métallique discontinu. Des pastilles de charbon actif entretiennent le vide à basse température.

\section{Joints froids.}

La démontabilité de l'enceinte à Hélium liquide contenant les enroulements supraconducteurs est une contrainte indispensable pour un cryostat d'essai. La géométrie annulaire imposée par l'existence d'un trou central débouchant introduit une double portée (Fig. 1). Seuls des joints « élastiques » peuvent assurer une étanchéité à une telle géométrie : des joints silicone (CAF 538) ont été essayés sur une enceinte d'essai. Nous avons pu vérifier que si la tenue à chaud d'un joint épais $(e>0,6 \mathrm{~mm})$ était mauvaise, elle était équivalente à celle d'un joint mince $(e<0,1 \mathrm{~mm})$ et tout à fait suffisante à basse température. Ces résultats ont été confirmés sur le cryostat définitif où des joints de ce type, ayant un diamètre de $280 \mathrm{~mm}$, ont été montés.

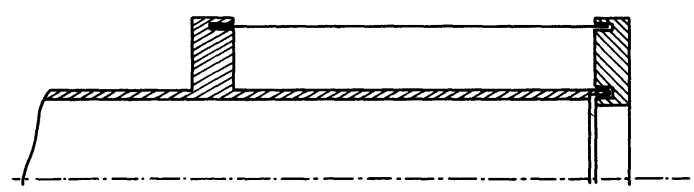

Fig. 1. - Géométrie schématique de l'enceinte Hélium.

[Schematic geometry of Helium vessel.]

\section{Description du cryostat (Fig. 2).}

L'enceinte Hélium est constituée de 2 tubes ( 6 et 7 ) fermés par flasques, l'un collé (12), l'autre démontable (5). Elle est maintenue par le tube (18) dont on a réduit les apports thermiques en thermalisant un point intermédiaire (17) relié par une tresse à l'échangeur en bronze fritté (15) et (16) qui récupère l'enthalpie des vapeurs d'He.

Pour éviter les vibrations éventuelles en cours de fonctionnement, l'enceinte est aussi supportée élastiquement par 6 tiges en acier inoxydable (3) fixées au cylindre extérieur. Pour réduire les apports thermiques par rayonnement radial, 2 écrans (8 et 9) sont thermalisés par conduction le long d'une nappe de fils de cuivre (Fig. 3), isolés entre eux et reliés à l'échangeur. Un excellent vide est maintenu entre les enceintes (1) et (2). Il est entretenu par du charbon actif (13). Les sorties de vapeur et les amenées de courant sont des tubes inox $(\varnothing 6 / 7 \mathrm{~mm})$ collés au cylindre (7) avec du stycast (EMERSON et CUMINGS 2350 FT + 24 LV).

La platine de cryostat porte l'alimentation en Hélium liquide dont la liaison amovible avec un siphon extérieur est assurée par un raccord Johnston, les différentes sorties des vapeurs et les fils d'instrumentation. Les sorties de courant des bobinages induits supraconducteurs, grâce à une boîte intermédiaire (24), peuvent être reliées soit à des amenées de courant classiques en cuivre (22), soit à une ligne de courant supraconductrice dans le cas où la machine serait reliée directement à un transformateur [7] supraconducteur.

\section{Essais cryogéniques.}

Plusieurs mises en froid ont été effectuées avec démontage complet de l'enceinte Hélium. Le joint silicone a toujours assuré une étanchéité parfaite à froid. La figure 4 présente une courbe caractéristique 


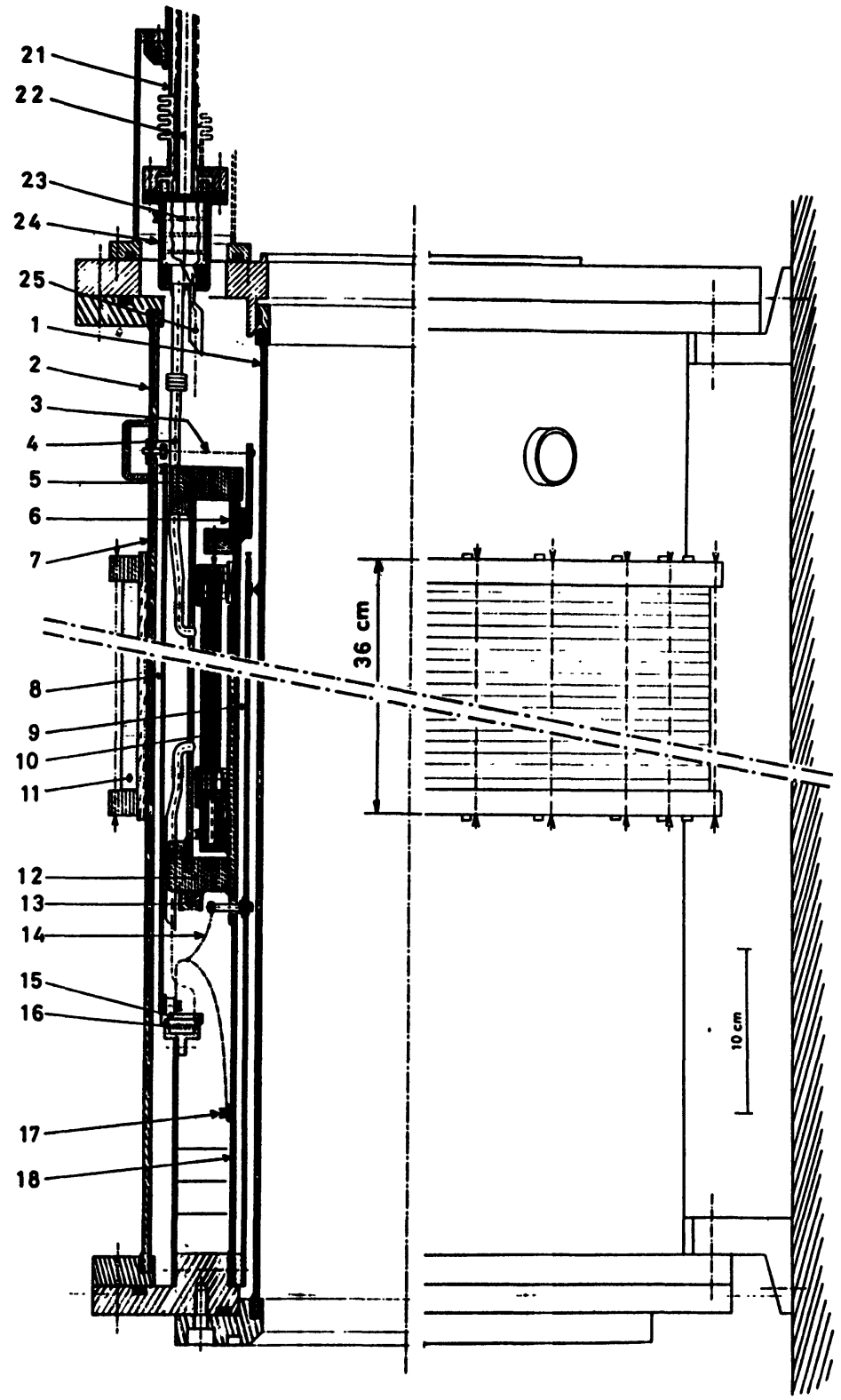

Fig. 2. - Coupe du cryostat statorique.

[Cross section of armature cryostat.]

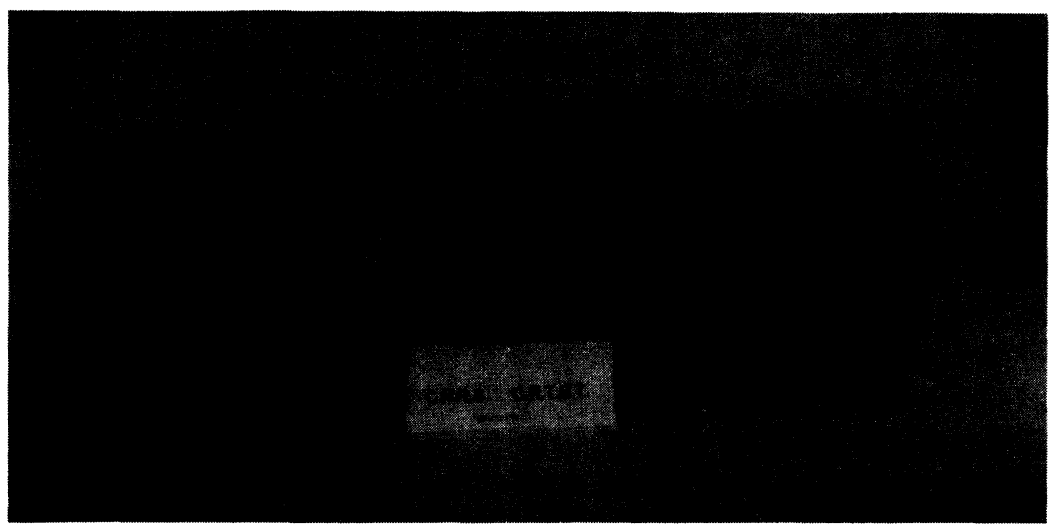

Fig. 3. - Nappe de fils de cuivre sur l'écran inférieur.

[Copper wires on the lower thermal schield.] 


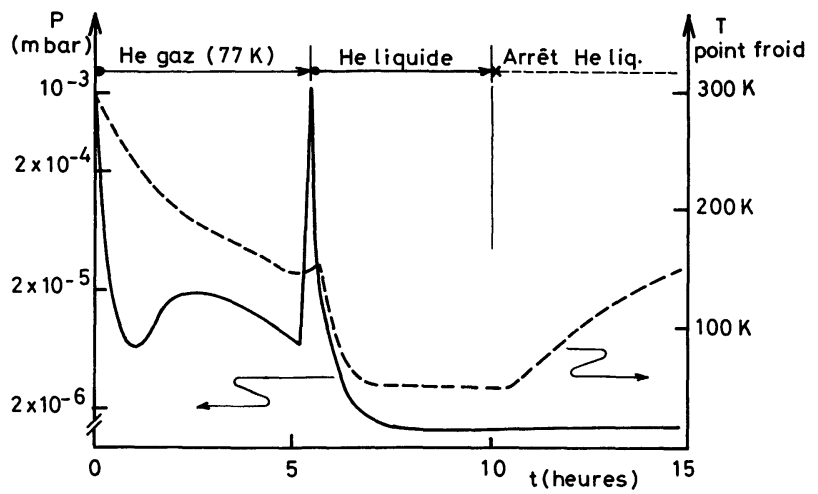

Fig. 4. - Evolution du vide et de la température du point froid au cours d'une mise en froid.

[Evolution of Vacuum and cold point temperature during a cooling-down.]

de la mise en froid et du fonctionnement à basse température : la courbe $P(t)$ illustre les propriétés de dégazage et de perméabilité variable avec la température des parois en composite époxy-fibre de verre. Le refroidissement est fait avec de l'Hélium gaz refroidi à la température de l'Azote liquide. L'Hélium liquide est injecté environ $6 \mathrm{~h}$ après le début de la mise en froid : après une nette détérioration du vide lors du changement du récipient d'alimentation, celui-ci s'améliore pour atteindre une valeur stable ( $\sim 2 \times 10^{-6}$ torr) qui se maintient en l'absence de tout pompage auxiliaire.

La température des écrans atteint $60 \mathrm{~K}$ et $75 \mathrm{~K}$. Les pertes du cryostat sont de $4 \mathrm{~W}$. Elles dépassent $10 \mathrm{~W}$ en siphonage continu, l'Hélium étant injecté $\mathrm{au}$ point bas du cryostat pour assurer un bon refroidissement de l'ensemble.

\section{Remerciements.}

Nous tenons à remercier Mrs Reynaud, Pilon et Leggeri pour l'aide technique qu'ils ont apportée à cette réalisation.

\section{Bibliographie}

[1] Dubois, P., Dubots, P., Février, A., Renard, J. C., GoYer, J. and KY, H. G., Ultra fine $\mathrm{NbTi}$ filaments wires for $\mathrm{AC}$ use, Helsinki ICEC 10 (1984).

[2] KhAlIL, A. and HAN, K. S., Mechanical and thermal properties of glass fiber reinforced composites at cryogenic temperatures. Adv. Cryog. Eng. vol. 28, p. 243, Ed. Timmerhauss (Plenum Press NY).

[3] Collins, E. W. and SMith, R. D., Specific heats of some cryogenic structural materials, $A d v$. Cryog. Eng. vol. 20, p. 290, Ed. Timmerhauss (Plenum Press NY).
[4] Schramm, R. E., Kasen, M. B., Fiber glass epoxy in a conical S.C. field magnet support, Adv. Cryog. Eng. vol. 24, p. 271, Ed. Timmerhauss (Plenum Press NY).

[5] KASEN, M. B., Mechanical an thermal properties of filamentary reinforced structural composites at cryogenic temperatures : glass reinforced composites, Cryogenics, p. 327 (June 1975).

[6] Hartwig, G., KNAack, S., Fibre epoxy composites at low temperatures, Cryogenics, p. 639 (Nov. 1984).

[7] SABRIE, J. L., Feasibility of large ac superconducting equipment, J. Phys. France 45 (1985) 717. 